\title{
Chemical Characterization of Lignin and Lipid Fractions in Industrial Hemp Bast Fibers Used for Manufacturing High-Quality Paper Pulps
}

\author{
Ana GutiÉRrez,* Isabel M. Rodríguez, And José C. Del Río \\ Instituto de Recursos Naturales y Agrobiología, CSIC, P.O. Box 1052, 41080 Seville, Spain
}

\begin{abstract}
The chemical composition of lignin and lipids of bast fibers from industrial hemp (Cannabis sativa) used for high-quality paper pulp production was studied. Pyrolysis-gas chromatography/mass spectrometry (Py-GC/MS) of fibers showed a lignin with a $p$-hydroxyphenyl:guaiacyl:syringyl unit $(\mathrm{H}$ : G:S) molar proportion of 13:53:34 (S/G ratio of 0.64 ). p-Hydroxycinnamic acids, namely, $p$-coumaric and ferulic acids, were found in only trace amounts. Among the lipids, the main compounds identified by GC/MS of the hemp fibers extracts were series of $n$-alkanes, free and esterified sterols and triterpenols, waxes, and long-chain $n$-fatty acids. Other compounds such as $n$-aldehydes, $n$-fatty alcohols, steroid hydrocarbons, and steroid and triterpenoid ketones as well as steryl glycosides were also found.
\end{abstract}

KEYWORDS: Industrial hemp; Cannabis sativa; bast fibers; paper pulp, lipids; lignin; steroids; pyrolysis

\section{INTRODUCTION}

Nonwood plants are the common fiber source for paper pulp production in developing countries where wood fibers are not available. In the developed world, although wood is still by far the main raw material for paper pulp manufacture, a market exists for high value-added papers from these fibers (1). Hemp (Cannabis sativa) is native to central Asia and has been cultivated in Asia and Europe for many years. In Europe, the cultivation of hemp declined in the 19th century but recently interest has been renewed, for example, in Germany, France, The Netherlands, the United Kingdom, Spain, and Italy (2). Uses for industrial hemp fibers in the paper industry mainly include specialty papers, that is, cigarette papers, Bible papers, and condenser papers (1).

Studies on the chemical composition of hemp bast fibers are important for optimizing the pulping and bleaching processes of this raw material. Among the several parameters that may affect pulp production are the composition of lignin and lipids in fibers. In general, the efficiency of pulping is directly proportional to the amount of syringyl (S) units in the lignin $(3,4)$. The guaiacyl $(G)$ units have a free $C-5$ position available for carbon-carbon interunit bonds, which make them fairly resistant to lignin depolymerization in pulping. On the other hand, it is well-known that lipophilic compounds present in raw materials cause significant environmental and technical problems in the manufacturing of paper pulp. During pulping, lipids are released from the fibers, forming colloidal pitch, which can deposit in either pulp or machinery and cause production troubles $(5-8)$. In the manufacture of alkaline pulps, a large

* Author to whom correspondence should be addressed (telephone +34-95-4624711; fax +34-95-4624002; e-mail anagu@irnase.csic.es). part of the lipids originally present in the raw material is removed during the cooking. However, some chemical species survive these processes and are found as pulp extractives, suspended in process waters or forming the so-called pitch deposits in circuits, equipment, and final product (9). Moreover, such extractives might contribute to the toxicity of paper pulp effluents and products (10).

To the best of the authors' knowledge, studies regarding the composition of lignin and lipids from industrial hemp fibers have been scarce. In the present study we have characterized the lignin in industrial hemp bast fibers using analytical pyrolysis coupled to gas chromatography/mass spectrometry (Py-GC/MS). $\mathrm{Py}-\mathrm{GC} / \mathrm{MS}$ is a powerful analytical tool for the rapid analysis of complex polymer mixtures including lignocellulosic materials $(11,12)$. It combines rapid thermal degradation and GC/MS analysis of marker compounds from pyrolytic breakdown. We also performed a detailed analysis of the chemical composition of lipids from industrial hemp bast fibers. These analyses were carried out by GC and GC/MS using high-temperature capillary columns of short and medium lengths, respectively (13). This method enables the elution and analysis of intact high molecular weight lipids such as waxes, sterol esters, and triglycerides.

\section{MATERIALS AND METHODS}

Samples. Industrial hemp (C. sativa) bast fibers (from Spain) were supplied by CELESA pulp mill (Tortosa, Spain). The retted hemp fibers contained $15 \%$ of core fibers. Hemp bast fibers were air-dried and subsequently milled using an Analysenmühle knife mill (Janke and Kunkel, Staufen, Germany). For the isolation of lipids, hemicellulose analysis, and Klason lignin content estimation, the milled samples were successively extracted with acetone ( $8 \mathrm{~h}$ in a Soxhlet apparatus) and hot water $\left(3 \mathrm{~h}\right.$ at $\left.100{ }^{\circ} \mathrm{C}\right)$. Klason lignin was estimated as the residue after sulfuric acid hydrolysis of the pre-extracted material, and neutral 


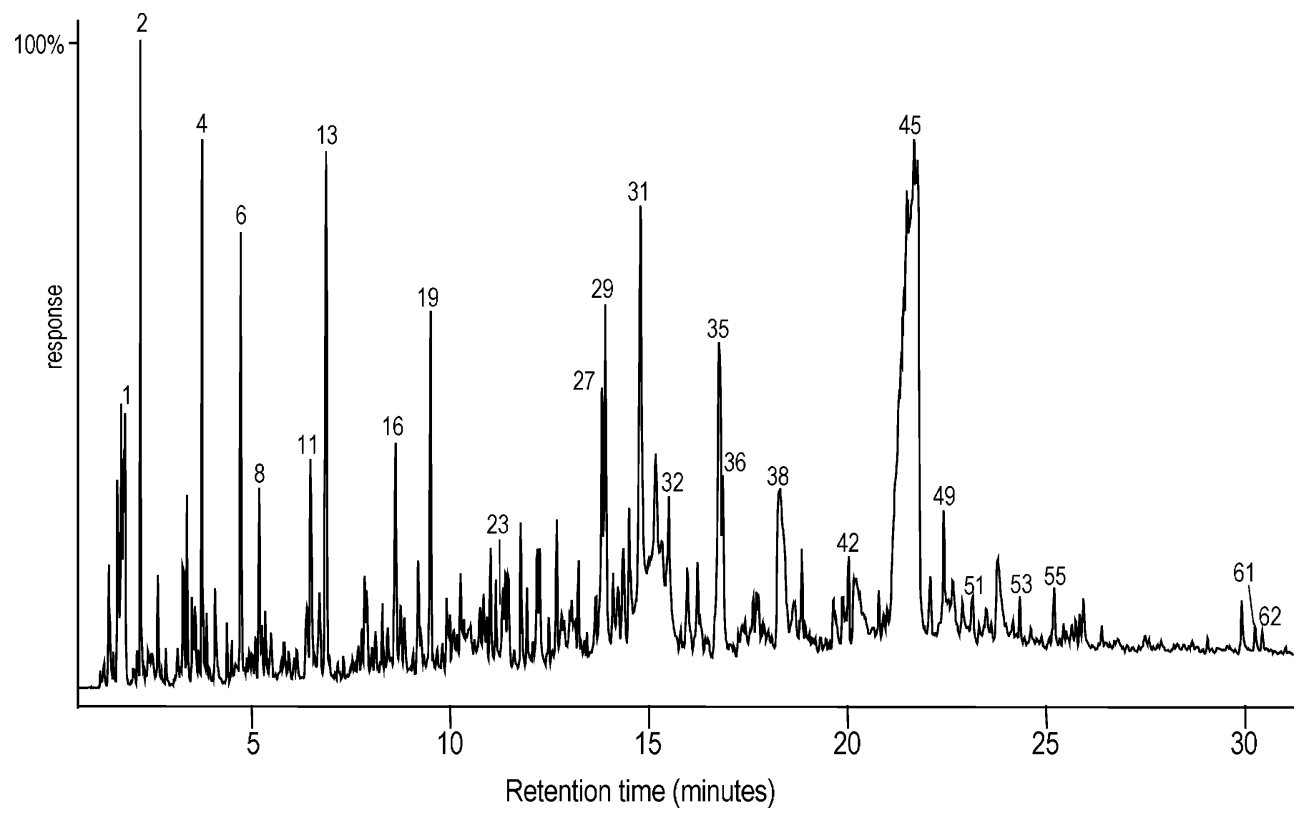

Figure 1. Py-GC/MS chromatogram of industrial hemp bast fibers. The identities and relative molar abundances of the compounds are listed in Table 1.

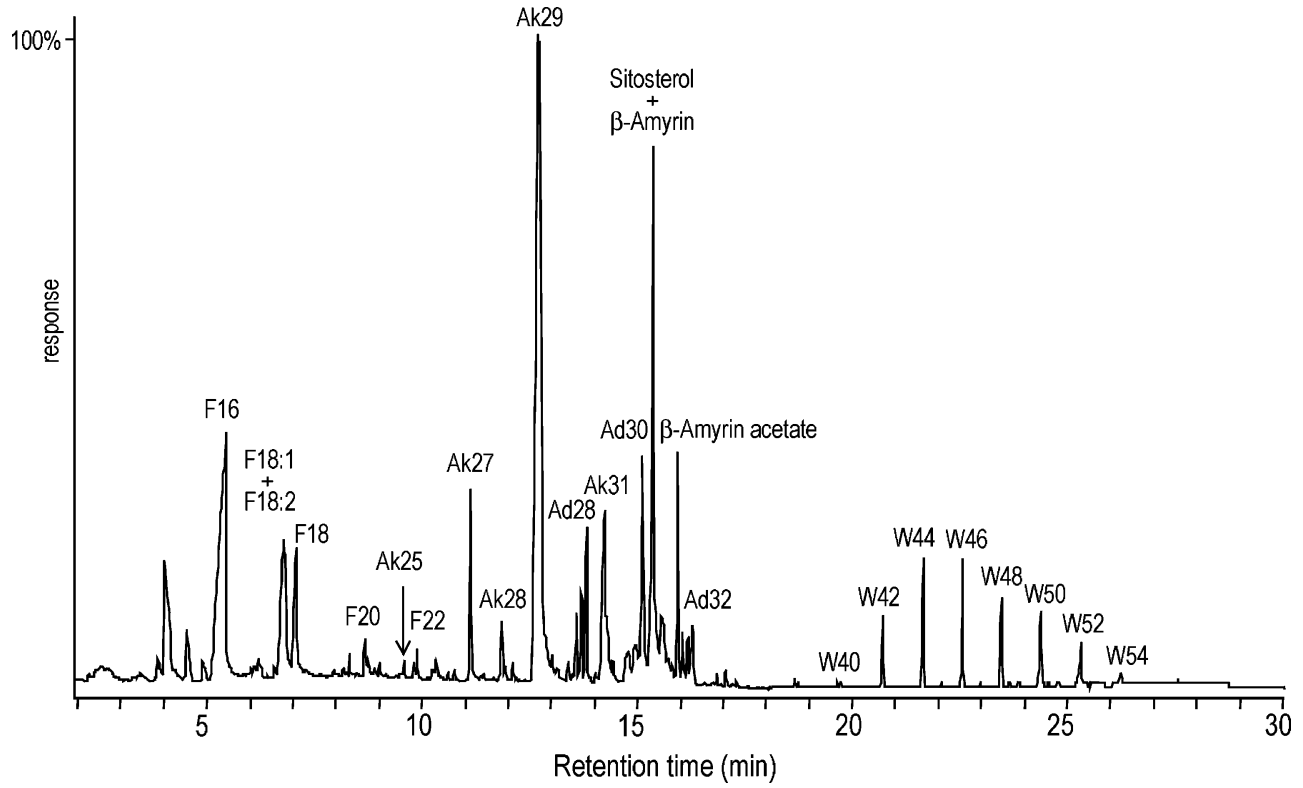

Figure 2. GC/MS chromatogram of the underivatized lipid extracts from industrial hemp bast fibers. $\mathrm{F}(n), n$-fatty acid series; Ak(n), $n$-alkane series; $\operatorname{Ad}(n)$, $n$-aldehyde series; $\mathrm{W}(n)$, wax series; $n$ denotes the total carbon atom number.

sugars from polysaccharide hydrolysis were analyzed as alditol acetates by GC according to Tappi rules T222 om- 88 and T249 om85 (14), respectively. Ash content was estimated as the residue after $6 \mathrm{~h}$ at 575 ${ }^{\circ} \mathrm{C}$. Two replicates were used for each sample. The composition (as percent of whole bast fiber) was as follows: ash, $2.0 \%$; acetone extractives, $0.5 \%$; water-soluble extract, $1.2 \%$; Klason lignin, $4.6 \%$; acid-soluble lignin, $1.5 \%$. The composition of neutral monosaccharides (as percent of total neutral carbohydrates) included rhamnose, $0.4 \%$; arabinose, $0.6 \%$; xylose, $1.0 \%$; mannose, $9.9 \%$; galactose, $1.6 \%$; and glucose, $86.4 \%$. No uronic acid determination was performed in this study. The composition of metals and other elements in the hemp fiber was analyzed by inductively coupled plasma spectrophotometry (ICPOES) after oxidation with concentrated $\mathrm{HNO}_{3}$ under pressure in a microwave digestor, with the following results: $\mathrm{Ca}, 5330 \mathrm{ppm}$; K, 2103 ppm; P, 667 ppm; Mg, 480 ppm; S, 372 ppm; Al, 241 ppm; Mn, 47 ppm; Na, 26 ppm; Si, 19 ppm; Sr, 17 ppm; Ba, 7 ppm; B, 7 ppm; Zn, $4 \mathrm{ppm}$. This study does not include nitrogen analysis. The acetone extracts were evaporated to dryness and redissolved in chloroform for chromatographic analysis of the lipophilic fraction.
GC and GC/MS Analyses. An HP 5890 gas chromatograph (Hewlett-Packard, Hoofddorp, The Netherlands) equipped with a splitsplitless injector and a flame ionization detector (FID) was used for $\mathrm{GC}$ analyses of the lipophilic compounds. The injector and the detector temperatures were set at 300 and $350{ }^{\circ} \mathrm{C}$, respectively. Samples were injected in the splitless mode. Helium was used as the carrier gas. The capillary column used was a $5 \mathrm{~m} \times 0.25 \mathrm{~mm}$ i.d., $0.1 \mu \mathrm{m}$, hightemperature, polyimide-coated fused silica tubing DB-5HT from J\&W Scientific (Folsom, CA), especially processed for use at $400{ }^{\circ} \mathrm{C}$. After a $1 \mathrm{~min}$ hold at $100{ }^{\circ} \mathrm{C}$, the oven was temperature-programmed from 100 to $350{ }^{\circ} \mathrm{C}$ at $15{ }^{\circ} \mathrm{C} / \mathrm{min}$, followed by a $3 \mathrm{~min}$ hold at $350{ }^{\circ} \mathrm{C}$. Peaks were quantified by area, and a mixture of standards (octadecane, palmitic acid, sitosterol, cholesteryl oleate, and campesteryl, stigmasteryl, and sitosteryl 3- $\beta$-D-glucopyranosides) was used to produce calibration curves. The data from the two replicates were averaged. In all cases the standard deviations from replicates were below $10 \%$ of the mean values.

The GC/MS analyses were performed on a model GC 8000 Top gas chromatograph (Thermo Finnigan, San Jose, CA) coupled to a 
Voyager quadrupole mass spectrometer detector (ThermoQuest Finnigan) equipped with a $15 \mathrm{~m} \times 0.25 \mathrm{~mm}$ i.d., $0.1 \mu \mathrm{m}$ DB-5HT fused silica capillary column $(\mathrm{J} \& \mathrm{~W})$. After an initial hold for $1 \mathrm{~min}$ at 120 ${ }^{\circ} \mathrm{C}$, the oven was heated from 120 to $380{ }^{\circ} \mathrm{C}$ at $10{ }^{\circ} \mathrm{C} / \mathrm{min}$, followed by a $5 \mathrm{~min}$ hold at $380^{\circ} \mathrm{C}$. The injector and transfer line temperatures were set at 300 and $350^{\circ} \mathrm{C}$, respectively. Helium was used as the carrier gas, and the injection was performed in splitless mode. Bis(trimethylsilyl)trifluoroacetamide (BSTFA) silylation was used when required. Compounds were identified by comparing their mass spectra with mass spectra in the Wiley and NIST libraries, by mass fragmentography, and, when possible, by comparison with authentic standards.

Py-GC/MS. The pyrolysis of hemp fibers was performed in duplicate with a Curie-point pyrolyzer (Horizon Instruments Ltd., Heathfield, Sussex, U.K.) coupled to a Varian Saturn 2000 GC/MS, using a $30 \mathrm{~m}$ $\times 0.25 \mathrm{~mm}$ i.d., $0.25 \mu \mathrm{m}$ DB-5 column. Approximately $100 \mu \mathrm{g}$ of finely divided sample was deposited on a ferromagnetic wire and then inserted into the glass liner and immediately placed in the pyrolyzer. The pyrolysis was carried out at $610^{\circ} \mathrm{C}$ for $4 \mathrm{~s}$. After a $1 \mathrm{~min}$ hold at 40 ${ }^{\circ} \mathrm{C}$, the chromatograph was programmed from 40 to $300{ }^{\circ} \mathrm{C}$ at a rate of $6^{\circ} \mathrm{C} / \mathrm{min}$. The final temperature was held for $20 \mathrm{~min}$. The injector, equipped with a liquid carbon dioxide cryogenic unit, was programmed from $-30{ }^{\circ} \mathrm{C}(1 \mathrm{~min})$ to $300{ }^{\circ} \mathrm{C}$ at $200{ }^{\circ} \mathrm{C} / \mathrm{min}$ while the GC/MS interface was kept at $300{ }^{\circ} \mathrm{C}$. For the pyrolysis in the presence of tetramethylammonium hydroxide (TMAH), to analyze $p$-hydroxycinnamic acids, $\sim 100 \mu \mathrm{g}$ of sample was mixed with $0.5 \mu \mathrm{L}$ of $25 \%$ TMAH. The wire was then inserted into the glass liner, which was subsequently placed in the pyrolyzer. The pyrolysis was carried out as described above. The compounds were identified by comparing the mass spectra obtained with those of the Wiley and NIST computer libraries and that reported in the literature $(11,12)$. Relative peak molar areas were calculated for carbohydrate and lignin pyrolysis products. The summed molar areas of the relevant peaks were normalized to $100 \%$, and the data for two repetitive pyrolysis experiments were averaged. The relative standard deviation for the pyrolysis data was $<10 \%$. No attempt was made to calculate the response factor for every single compound released. However, for most of the lignin-derived phenols, the response factors are nearly identical (15), with the exception of vanillin, but this is a minor peak here.

\section{RESULTS AND DISCUSSION}

The hemp fiber was characterized by its low lignin content, estimated as Klason lignin, which accounted for $4.6 \%$ of the total fiber. This value is lower than those of other nonwood bast fibers such as kenaf but similar to that of flax $(16,17)$. The lipid content, $\sim 0.5 \%$, is also low and similar to that of kenaf but lower than those of other nonwood materials used for papermaking, such as flax $(18,19)$. On the other hand, the hemicellulose fraction was mainly constituted by mannose. Finally, the ash content, $2 \%$, is also low, and the composition of the different metals and other elements in the hemp fibers revealed a predominance of $\mathrm{Ca}$ and $\mathrm{K}$ and very low contents of other elements. It is important to note the low amounts of silica found in the hemp fiber compared to those of other nonwood fibers used for papermaking, such as wheat straw.

Lignin Composition. The low lignin content of the hemp fibers is, in principle, advantageous for its use in paper pulp manufacturing as it would require fewer chemicals and less drastic conditions during pulping. However, it is known that the lignin composition also strongly affects pulping $(3,4)$. To analyze in situ the chemical composition of lignin, the hemp bast fibers were subjected to Py-GC/MS. The Py-GC/MS chromatogram is shown in Figure 1, and the identities and relative abundances of the released compounds are listed in Table 1. The Py-GC/MS analysis of the hemp fibers released predominantly compounds arising from carbohydrates and very minor amounts of lignin-derived phenols, in agreement with the relatively low lignin content estimated as Klason lignin. As
Table 1. Identification and Relative Molar Abundance of Compounds Released after Py-GC/MS of Industrial Hemp Bast Fibers ${ }^{a}$

\begin{tabular}{|c|c|c|c|c|}
\hline no. & compound & $\begin{array}{l}\text { mass fragments } \\
\qquad(\mathrm{m} / \mathrm{z})\end{array}$ & origin & $\begin{array}{l}\text { abundance } \\
(\%)\end{array}$ \\
\hline 1 & hydroxyacetaldehyde & $42 / 60$ & C & 8.1 \\
\hline 2 & 3-hydroxypropanal & $73 / \overline{74}$ & $\mathrm{C}$ & 4.2 \\
\hline 3 & $(3 H)$-furan-2-one & $5 5 \longdiv { 8 4 }$ & C & 0.5 \\
\hline 4 & 2,3-butanedione & $56 / 57 / 86$ & $\mathrm{C}$ & 4.1 \\
\hline 5 & $(2 H)$-furan-3-one & $55 / 84^{-}$ & $\mathrm{C}$ & 0.9 \\
\hline 6 & 2-furaldehyde & $67 / \overline{95} / 96$ & $\mathrm{C}$ & 3.0 \\
\hline 7 & 2-methylfuran & $53 / 81 / 82$ & $\mathrm{C}$ & 0.5 \\
\hline 8 & 2-(hydroxymethyl)furan & $43 / 70 / 81 / 98$ & C & 1.5 \\
\hline 9 & cyclopent-1-ene-3,4-dione & $54 / 68 / 96$ & $\mathrm{C}$ & 0.4 \\
\hline 10 & 4-methyltetrahydrofuran-3-one & $43 / 72^{-0}$ & $\mathrm{C}$ & 0.2 \\
\hline 11 & $(5 H)$-furan-2-one & $\overline{55 / 84}$ & $\mathrm{C}$ & 2.3 \\
\hline 12 & acetylfuran & $\overline{43} / 95 / 110$ & C & 0.2 \\
\hline 13 & 2,3-dihydro-5-methylfuran-2-one & $55 / 69 / 98$ & $\mathrm{C}$ & 6.0 \\
\hline 14 & 5-methyl-2-furfuraldehyde & $53 / 109 / 110$ & $\mathrm{C}$ & 1.0 \\
\hline 15 & phenol & $65 / 66 / 94$ & $\mathrm{LH}$ & 0.4 \\
\hline 16 & 5,6-dihydropyran-2,5-dione & $68 / 98^{-}$ & $\mathrm{C}$ & 2.4 \\
\hline 17 & $\begin{array}{l}\text { 4-hydroxy-5,6-dihydro-(2H)- } \\
\text { pyran-2-one }\end{array}$ & $\overline{58} / 85 / 114$ & $\mathrm{C}$ & 0.5 \\
\hline 18 & $\begin{array}{l}\text { 3-hydroxy-2-methyl-2-cyclo- } \\
\text { penten-1-one }\end{array}$ & $55 / 84 / 112$ & C & 0.9 \\
\hline 19 & $\begin{array}{l}\text { 2-hydroxy-3-methyl-2-cyclo- } \\
\text { penten-1-one }\end{array}$ & $55 / 84 / 112$ & C & 2.7 \\
\hline 20 & 2,3-dimethylcyclopenten-1-one & $67 / 95 / 110$ & C & 0.2 \\
\hline 21 & 4-methylphenol & $77 / 107 / 108$ & $\mathrm{LH}$ & 0.4 \\
\hline 22 & 2-furoic acid, methyl ester & $6 7 \longdiv { 9 5 / 1 2 6 }$ & $\mathrm{C}$ & 0.8 \\
\hline 23 & guaiacol & $81 / 109 / 124$ & LG & 0.5 \\
\hline 24 & 4-ethylphenol & $77 / 107 / 122$ & $\mathrm{LH}$ & 0.1 \\
\hline 25 & 3,4-dihydroxybenzaldehyde & $81 / 109 / 137 / 138$ & LM & 0.4 \\
\hline 26 & $\begin{array}{l}\text { 3,5-dihydroxy-2-methyl-(4H)- } \\
\text { pyran-4-one }\end{array}$ & $70 / 98 / 1 \overline{26}$ & $\mathrm{C}$ & 0.3 \\
\hline 27 & $\begin{array}{l}\text { 5-hydroxymethyl-2-tetrahydro- } \\
\text { furaldehyde-3-one }\end{array}$ & 43/57/69/70/85 & C & 1.5 \\
\hline 28 & 4-methylguaiacol & $95 / 123 / 138$ & LG & 0.3 \\
\hline 29 & catechol & $64 / 81 / 92 / 110$ & $\mathrm{LM} / \mathrm{C}$ & 3.9 \\
\hline 30 & $\begin{array}{l}\text { 5-hydroxymethyl-2-furaldehyde } \\
\text { (isomer) }\end{array}$ & $69 / 97 / 109 / 126$ & C & 0.5 \\
\hline 31 & 5-hydroxymethyl-2-furaldehyde & $69 / 97 / 109 / 126$ & C & 5.1 \\
\hline 32 & 3-methoxycatechol & $79 / \overline{97} / 125 / 140$ & LM & 0.9 \\
\hline 33 & 4-ethylguaiacol & $122 / 137 / 152$ & LG & 0.2 \\
\hline 34 & 4-methylcatechol & $78 / 1 \overline{07 / 123 / 124}$ & LM & 0.5 \\
\hline 35 & $\begin{array}{l}\text { 1,4-dideoxy-D-glycerohex-1-ene- } \\
\text { pyrenone-3-ulose }\end{array}$ & 43/73/87/113/144 & $\mathrm{C}$ & 3.4 \\
\hline 36 & 4-vinylguaiacol & $107 / 135 / 150$ & LG & 1.3 \\
\hline 37 & syringol & $111 / \overline{139} / 154$ & LS & 0.2 \\
\hline 38 & pyrogallol & $52 / 80 / 97 / 108 / 126$ & LM & 4.8 \\
\hline 39 & 1,6-anhydrogalactopyranose & $57 / 60 / 73 / 98$ & $\mathrm{C}$ & 0.3 \\
\hline 40 & vanillin & $109 / 151 / 152$ & LG & 0.6 \\
\hline 41 & 4-methylsyringol & $125 / 153 / \overline{168}$ & LS & 0.1 \\
\hline 42 & trans-isoeugenol & $131 / 149 / \overline{164}$ & $L G$ & 0.4 \\
\hline 43 & homovanillin & $122 / 137 / \overline{166}$ & LG & 0.2 \\
\hline 44 & 1,6-anhydromannopyranose & $57 / 6 \overline{0 / 73 / 98}$ & C & 0.9 \\
\hline 45 & $\begin{array}{l}\text { 1,6-anhydroglucopyranose } \\
\text { (levoglucosan) }\end{array}$ & $57 / 60 / 73 / 98$ & $\mathrm{C}$ & 28.7 \\
\hline 46 & 4-ethylsyringol & $167 / 182$ & LS & 0.5 \\
\hline 47 & guaiacylacetone & $122 / 137 / 180$ & LG & 0.2 \\
\hline 48 & 1,6 -anhydro- $\beta$-D-glucofuranose & $73 / 8 \overline{5 / 115}$ & C & 0.4 \\
\hline 49 & 4-vinylsyringol & $\overline{13} 7 / 165 / 180$ & LS & 0.6 \\
\hline 50 & guaiacyl vinyl ketone & $123 / 151 / 178$ & LG & 0.2 \\
\hline 51 & 4-allylsyringol & $167 / 179 / 194$ & LS & 0.3 \\
\hline 52 & cis-4-propenylsyringol & $167 / 179 / \overline{194}$ & LS & 0.1 \\
\hline 53 & syringaldehyde & $167 / 181 / \longdiv { 1 8 2 }$ & LS & 0.2 \\
\hline 54 & cis-coniferyl alcohol & $124 / 137 / 151 / 180$ & LG & 0.1 \\
\hline 55 & trans-4-propenylsyringol & $167 / 179 / 194$ & LS & 0.4 \\
\hline 56 & acetosyringone & $153 / 181 / \longdiv { 1 9 6 }$ & LS & 0.1 \\
\hline 57 & trans-coniferaldehyde & $107 / 135 / 147 / 178$ & LG & 0.1 \\
\hline 58 & trans-coniferyl alcohol & $124 / 137 / 151 / 180$ & LG & 0.3 \\
\hline 59 & syringylacetone & $123 / 167 / 210$ & LS & 0.1 \\
\hline 60 & cis-sinapyl alcohol & $154 / 167 / 210$ & LS & 0.1 \\
\hline 61 & trans-sinapaldehyde & $137 / 165 / 180 / 208$ & LS & 0.1 \\
\hline 62 & trans-sinapyl alcohol & $154 / 167 / 210^{-}$ & LS & 0.1 \\
\hline & $\% \mathrm{H}$ & & & 12.8 \\
\hline & $\% G$ & & & 53.0 \\
\hline & $\% S$ & & & 34.2 \\
\hline & $S / G$ & & & 0.64 \\
\hline
\end{tabular}

${ }^{a} \mathrm{C}$, carbohydrates; LM, modified lignin; LH, $p$-hydroxyphenyl lignin units, $H$; LG, guaiacyl lignin units, G; LS, syringyl lignin units, $S$. Underlined mass fragments indicate base peaks. 
Table 2. Composition and Abundance of Lipids from Industrial Hemp Bast Fibers (Main Mass Fragments Are Included)

\begin{tabular}{|c|c|c|c|c|c|}
\hline compound & mass fragments & $\begin{array}{l}\text { abundance } \\
\text { (mg/100 g) }\end{array}$ & compound & mass fragments & $\begin{array}{l}\text { abundance } \\
\text { (mg/100 g) }\end{array}$ \\
\hline$n$-alkanes & & 52.50 & triterpenoid/steroid ketones & & 8.28 \\
\hline$n$-heneicosane & $57 / 71 / 85 / 296$ & 0.16 & $\beta$-amyrenone & $189 / 203 / 218 / 409 / 424$ & 1.91 \\
\hline$n$-tricosane & $57 / 71 / 85 / 324$ & 0.12 & $\alpha$-amyrenone & $189 / 203 / 218 / 409 / 424$ & 0.38 \\
\hline$n$-pentacosane & $57 / 71 / 85 / 352$ & 0.31 & stigmastan-3-one & $231 / 232 / 414$ & 0.84 \\
\hline$n$-hexacosane & $57 / 71 / 85 / 366$ & 0.19 & stigmasta-7,22-dien-3-one & $55 / 269 / 298 / 367 / 410$ & 0.61 \\
\hline$n$-heptacosane & $57 / 71 / 85 / 380$ & 4.71 & stigmasta-3,5-dien-7-one & $174 / 269 / 410$ & 0.15 \\
\hline n-octacosane & $57 / 71 / 85 / 394$ & 1.25 & stigmast-4-en-3-one & $124 / 229 / 412$ & 2.52 \\
\hline$n$-nonacosane & $57 / 71 / 85 / 408$ & 39.03 & stigmastadienone isomer & $\overline{57 / 136 / 174 / 269 / 410}$ & 0.64 \\
\hline$n$-triacontane & $57 / 71 / 85 / 422$ & 0.92 & ergostane-3,6-dione & $137 / 245 / 414$ & 0.09 \\
\hline$n$-hentriacontane & $57 / 71 / 85 / 436$ & 4.72 & stigmast-4-en-3,6-dione & $137 / 398 / 408 / 411 / 426$ & 0.03 \\
\hline$n$-tritriacontane & $57 / 71 / 85 / 464$ & 1.09 & stigmastane-3,6-dione & $245 / 287 / 428$ & 1.11 \\
\hline fatty acids & & 21.66 & waxes & & 21.34 \\
\hline n-tetradecanoic acid & $60 / 73 / 129 / 228$ & 0.25 & wax $\mathrm{C}_{40}$ & $57 / 257 / 285 / 313 / 592$ & 0.10 \\
\hline$n$-pentadecanoic acid & $60 / \overline{73} / 129 / 242$ & 0.29 & wax $\mathrm{C}_{42}$ & $57 / 313 / 341 / 353 / 620$ & 1.95 \\
\hline 9-hexadecenoic acid & $55 / 69 / 236 / 254$ & 0.05 & wax $\mathrm{C}_{44}$ & $57 / 257 / 313 / 341 / 369 / 648$ & 4.23 \\
\hline$n$-hexadecanoic acid & $60 / 73 / 129 / 256$ & 11.15 & wax $\mathrm{C}_{45}$ & $57 / 313 / 355 / 369 / 662$ & 0.12 \\
\hline 9,12-octadecadienoic acid & $67 / 81 / 280$ & 3.82 & wax $\mathrm{C}_{46}$ & $57 / 257 / 313 / 341 / 369 / 676$ & 4.47 \\
\hline 9-octadecenoic acid & $75569 / 264$ & 2.27 & wax $\mathrm{C}_{47}$ & $57 / 313 / 341 / 369 / 383 / 690$ & 0.09 \\
\hline$n$-octadecanoic acid & $6 \overline{0 / 73 / 129 / 284}$ & 2.10 & wax $\mathrm{C}_{48}$ & $57 / 313 / 341 / 369 / 397 / 704$ & 3.25 \\
\hline$n$-eicosanoic acid & $60 / 73 / 129 / 312$ & 0.78 & wax $\mathrm{C}_{49}$ & $57 / 313 / 327 / 341 / 369 / 718$ & 0.13 \\
\hline$n$-docosanoic acid & $60 / \overline{73} / 129 / 340$ & 0.48 & wax $C_{50}$ & $57 / 341 / 369 / 437 / 453 / 760$ & 3.66 \\
\hline$n$-tetracosanoic acid & $60 / 73 / 129 / 368$ & 0.23 & wax $\mathrm{C}_{51}$ & $57 / 341 / 369 / 397 / 746$ & 0.14 \\
\hline$n$-hexacosanoic acid & $60 / 73 / 129 / 396$ & 0.07 & wax $\mathrm{C}_{52}$ & $57 / 341 / 369 / 437 / 435 / 760$ & 2.61 \\
\hline n-octacosanoic acid & $60 / 73 / 129 / 424$ & 0.08 & wax $\mathrm{C}_{54}$ & $57 / 369 / 397 / 425 / 788$ & 0.59 \\
\hline$n$-triacontanoic acid & $60 / 73 / 129 / 452$ & 0.09 & sterol/triterpenol esters & & 26.50 \\
\hline$\alpha$-hydroxy fatty acids & & 0.43 & $\beta$-amyrin acetate & $189 / 203 / 218 / 468$ & 17.68 \\
\hline 2-hydroxyhexadecanoic acid & $73 / 299 / 343^{a}$ & 0.31 & $\alpha$-amyrin acetate & $189 / 203 / 218 / 468$ & 4.43 \\
\hline 2-hydroxydocosanoic acid & $\overline{73} / 383 / 427^{a}$ & 0.03 & taraxerol acetate & $204 / 218 / \overline{426}$ & 0.80 \\
\hline 2-hydroxytricosanoic acid & $73 / 397 / 441^{a}$ & 0.03 & lupeol acetate & $43 / 189 / 218 / 468$ & 2.64 \\
\hline 2-hydroxytetracosanoic acid & $\overline{73} / 411 / 455^{a}$ & 0.05 & sitosterol hexadecanoate & $\overline{147 / 381 / 396}$ & 0.04 \\
\hline 2-hydroxypentacosanoic acid & $73 / 425 / 469^{a}$ & 0.01 & sitosterol octadecanoate & $1 4 7 / 3 8 1 \longdiv { 3 9 6 }$ & 0.01 \\
\hline fatty alcohols & & 6.01 & $\beta$-amyrin tetradecanoate & $1 8 9 / 2 0 3 \longdiv { 2 1 8 }$ & 0.04 \\
\hline$n$-octadecanol & $75 / 103 / 327^{b}$ & 0.19 & $\beta$-amyrin pentadecanoate & $1 8 9 / 2 0 3 \longdiv { 2 1 8 }$ & 0.05 \\
\hline$n$-eicosanol & $75 / 103 / 355^{b}$ & 0.33 & $\beta$-amyrin hexadecanoate & $1 8 9 / 2 0 3 \longdiv { 2 1 8 }$ & 0.15 \\
\hline$n$-docosanol & $7 5 / 1 0 3 \longdiv { 3 _ { 8 3 } } b$ & 1.04 & $\beta$-amyrin octadecanoate & $1 8 9 / 2 0 3 \longdiv { 2 1 8 }$ & 0.03 \\
\hline$n$-tetracosanol & $75 / 103 / \overline{411^{b}}$ & 0.33 & $\beta$-amyrin eicosanoate & $189 / 203 / \overline{218}$ & 0.06 \\
\hline$n$-hexacosanol & $75 / 103 / \overline{439} b$ & 0.59 & $\beta$-amyrin docosanoate & $189 / 203 / 218$ & 0.10 \\
\hline$n$-octacosanol & $75 / 103 / \overline{467} b$ & 1.57 & $\beta$-amyrin tetracosanoate & $189 / 203 / \overline{218}$ & 0.20 \\
\hline$n$-triacontanol & $75 / 103 / \overline{495} b$ & 1.63 & $\beta$-amyrin hexacosanoate & $189 / 203 / \overline{218}$ & 0.10 \\
\hline$n$-dotriacontanol & $75 / 103 / \overline{523}^{b}$ & 0.33 & $\beta$-amyrin octacosanoate & $1 8 9 / 2 0 3 \longdiv { 2 1 8 }$ & 0.04 \\
\hline aldehydes & & 7.91 & $\beta$-amyrin triacontanoate & $1 8 9 / 2 0 3 \longdiv { 2 1 8 }$ & 0.01 \\
\hline$n$-heneicosanal & $82 / 96 / 292$ & 0.09 & $\alpha$-amyrin tetradecanoate & $1 8 9 / 2 0 3 \longdiv { 2 1 8 }$ & $<0.01$ \\
\hline$n$-docosanal & $\overline{82} / 96 / 306$ & 0.11 & $\alpha$-amyrin hexadecanoate & $1 8 9 / 2 0 3 \longdiv { 2 1 8 }$ & 0.02 \\
\hline$n$-tricosanal & $82 / 96 / 320$ & 0.18 & $\alpha$-amyrin octadecanoate & $1 8 9 / 2 0 3 \longdiv { 2 1 8 }$ & 0.01 \\
\hline$n$-tetracosanal & $\overline{82} / 96 / 334$ & 0.08 & $\alpha$-amyrin eicosanoate & $1 8 9 / 2 0 3 \longdiv { 2 1 8 }$ & 0.02 \\
\hline$n$-pentacosanal & $\overline{82} / 96 / 348$ & 0.05 & $\alpha$-amyrin docosanoate & $1 8 9 / 2 0 3 \longdiv { 2 1 8 }$ & 0.02 \\
\hline$n$-hexacosanal & $82 / 96 / 362$ & 0.39 & $\alpha$-amyrin tetracosanoate & $189 / 203 / \overline{218}$ & 0.02 \\
\hline n-octacosanal & $82 / 96 / 390$ & 2.51 & $\alpha$-amyrin hexacosanoate & $1 8 9 / 2 0 3 \longdiv { 2 1 8 }$ & 0.02 \\
\hline$n$-nonacosanal & $82 / 96 / 404$ & 0.15 & $\alpha$-amyrin octacosanoate & $189 / 203 / \overline{218}$ & $<0.01$ \\
\hline$n$-triacontanal & $82 / 96 / 418$ & 3.72 & $\alpha$-amyrin triacontanoate & $189 / 203 / 218$ & $<0.01$ \\
\hline$n$-dotriacontanal & $82 / 96 / 446$ & 0.63 & steryl glycosides & & 1.80 \\
\hline steroid hydrocarbons & & 2.84 & campesteryl 3- $\beta$-D-gluco- & $204 / 217 / 361 / 383^{b}$ & 0.15 \\
\hline stigmasta-3,5,22-triene & $135 / 143 / 394$ & 1.46 & pyranoside & & \\
\hline stigmasta-3,5-diene & $\overline{81 / 147 / 381 / 396}$ & 1.38 & stigmasteryl 3- $\beta$-D-gluco- & $204 / 217 / 361 / 395^{b}$ & 0.14 \\
\hline free sterols/triterpenols & & 31.81 & pyranoside & & \\
\hline campesterol & $55 / 145 / 213 / 382 / 400$ & 1.70 & sitosteryl 3- $\beta$-D-gluco- & $204 / 217 / 361 / 397^{b}$ & 1.51 \\
\hline stigmasterol & $55 / 83 / 255 / 394 / 412$ & 1.85 & pyranoside & & \\
\hline sitosterol & $145 / 213 / 396 / 414$ & 13.93 & & & \\
\hline stigmastanol & $2 1 5 \longdiv { 4 1 6 }$ & 3.78 & & & \\
\hline$\beta$-amyrin & $203 / 218 / 426$ & 9.47 & & & \\
\hline$\alpha$-amyrin & $203 / 218 / 426$ & 1.08 & & & \\
\hline$\alpha$-tocopherol & $165 / 205 / 430$ & 1.40 & & & \\
\hline
\end{tabular}

\footnotetext{
${ }^{a}$ As methyl ester and TMS ether derivatives. ${ }^{b}$ As TMS ether derivatives. Underlined mass fragments indicate base peaks.
}

a result, carbohydrate pyrolysis products represented $81 \%$ on average and phenols from lignin represented $19 \%$ of the total identified compounds from hemp fibers. The main carbohydratederived compounds were hydroxyacetaldehyde (1), 3-hydroxypropanal (2), 2-furaldehyde (6), 2,3-dihydro-5-methylfuran-2one (13), 2-hydroxy-3-methyl-2-cyclopenten-1-one (19), 5-hydroxymethyl-2-furaldehyde (31), 1,4-dideoxy-D-glycerohex-1- enopyranone-3-ulose (35), and levoglucosan (45), the latter being the most prominent peak in the pyrogram. Among the lignin-derived compounds, the pyrogram of hemp bast fibers showed compounds derived from $p$-hydroxyphenyl $(\mathrm{H})$, guaiacyl $(\mathrm{G})$, and syringyl (S) lignin units, with a predominance of the $\mathrm{G}$ units. The main lignin-derived compound identified was 4-vinylguaiacol (36). Other guaiacyl counterparts including 
Table 3. Composition and Abundance of the Different Individual Esters Identified among the Waxes Identified in Industrial Hemp Fibers

\begin{tabular}{|c|c|c|}
\hline waxes & $\begin{array}{l}\text { fatty acid: } \\
\text { fatty alcohol }\end{array}$ & $\begin{array}{l}\text { abundance } \\
\text { (mg/100 g) }\end{array}$ \\
\hline \multicolumn{3}{|l|}{ wax $\mathrm{C}_{40}$} \\
\hline tetradecanoic acid, hexacosyl ester & $\mathrm{C}_{14}: \mathrm{C}_{26}$ & 0.02 \\
\hline hexadecanoic acid, tetracosyl ester & $\mathrm{C}_{16}: \mathrm{C}_{24}$ & 0.02 \\
\hline octadecanoic acid, docosyl ester & $\mathrm{C}_{18}: \mathrm{C}_{22}$ & 0.06 \\
\hline \multicolumn{3}{|l|}{ wax $\mathrm{C}_{42}$} \\
\hline hexadecanoic acid, hexacosyl ester & $\mathrm{C}_{16}: \mathrm{C}_{26}$ & 0.11 \\
\hline octadecanoic acid, tetracosyl ester & $\mathrm{C}_{18}: \mathrm{C}_{24}$ & 0.04 \\
\hline eicosanoic acid, docosyl ester & $\mathrm{C}_{20}: \mathrm{C}_{22}$ & 1.44 \\
\hline docosanoic acid, eicosyl ester & $\mathrm{C}_{22}: \mathrm{C}_{20}$ & 0.33 \\
\hline tetracosanoic acid, octadecyl ester & $\mathrm{C}_{24}: \mathrm{C}_{18}$ & 0.03 \\
\hline \multicolumn{3}{|l|}{ wax $\mathrm{C}_{44}$} \\
\hline hexadecanoic acid, octacosyl ester & $\mathrm{C}_{16}: \mathrm{C}_{28}$ & 0.28 \\
\hline octadecanoic acid, hexacosyl ester & $\mathrm{C}_{18}: \mathrm{C}_{26}$ & 0.09 \\
\hline eicosanoic acid, tetracosyl ester & $\mathrm{C}_{20}: \mathrm{C}_{24}$ & 0.98 \\
\hline docosanoic acid, docosyl ester & $\mathrm{C}_{22}: \mathrm{C}_{22}$ & 2.40 \\
\hline tetracosanoic acid, eicosyl ester & $\mathrm{C}_{24}: \mathrm{C}_{20}$ & 0.45 \\
\hline hexacosanoic acid, octadecyl ester & $\mathrm{C}_{26}: \mathrm{C}_{18}$ & 0.03 \\
\hline \multicolumn{3}{|l|}{ wax $\mathrm{C}_{45}$} \\
\hline hexadecanoic acid, nonacosyl ester & $\mathrm{C}_{16}: \mathrm{C}_{29}$ & 0.01 \\
\hline eicosanoic acid, pentacosyl ester & $\mathrm{C}_{20}: \mathrm{C}_{25}$ & 0.05 \\
\hline docosanoic acid, tricosyl ester & $\mathrm{C}_{22}: \mathrm{C}_{23}$ & 0.03 \\
\hline tetracosanoic acid, heneicosyl ester & $\mathrm{C}_{24}: \mathrm{C}_{21}$ & 0.03 \\
\hline \multicolumn{3}{|l|}{ wax $\mathrm{C}_{46}$} \\
\hline hexadecanoic acid, triacontyl ester & $\mathrm{C}_{16}: \mathrm{C}_{30}$ & 0.54 \\
\hline octadecanoic acid, octacosyl ester & $\mathrm{C}_{18}: \mathrm{C}_{28}$ & 0.24 \\
\hline eicosanoic acid, hexacosyl ester & $\mathrm{C}_{20}: \mathrm{C}_{26}$ & 1.75 \\
\hline docosanoic acid, tetracosyl ester & $\mathrm{C}_{22}: \mathrm{C}_{24}$ & 1.50 \\
\hline tetracosanoic acid, docosyl ester & $\mathrm{C}_{24}: \mathrm{C}_{22}$ & 0.28 \\
\hline hexacosanoic acid, eicosyl ester & $\mathrm{C}_{26}: \mathrm{C}_{20}$ & 0.14 \\
\hline octacosanoic acid, octadecyl ester & $\mathrm{C}_{28}: \mathrm{C}_{18}$ & 0.02 \\
\hline \multicolumn{3}{|l|}{ wax $\mathrm{C}_{47}$} \\
\hline hexadecanoic acid, hentriacontyl ester & $\mathrm{C}_{16}: \mathrm{C}_{31}$ & 0.01 \\
\hline eicosanoic acid, heptacosyl ester & $\mathrm{C}_{20}: \mathrm{C}_{27}$ & 0.03 \\
\hline docosanoic acid, pentacosyl ester & $\mathrm{C}_{22}: \mathrm{C}_{25}$ & 0.05 \\
\hline \multicolumn{3}{|l|}{ wax $\mathrm{C}_{48}$} \\
\hline hexadecanoic acid, dotriacontyl ester & $\mathrm{C}_{16}: \mathrm{C}_{32}$ & 0.11 \\
\hline octadecanoic acid, triacontyl ester & $\mathrm{C}_{18}: \mathrm{C}_{30}$ & 0.13 \\
\hline eicosanoic acid, octacosyl ester & $\mathrm{C}_{20}: \mathrm{C}_{28}$ & 1.18 \\
\hline docosanoic acid, hexacosyl ester & $\mathrm{C}_{22}: \mathrm{C}_{26}$ & 0.87 \\
\hline tetracosanoic acid, tetracosyl ester & $\mathrm{C}_{24}: \mathrm{C}_{24}$ & 0.49 \\
\hline hexacosanoic acid, docosyl ester & $\mathrm{C}_{26}: \mathrm{C}_{22}$ & 0.39 \\
\hline octacosanoic acid, eicosyl ester & $\mathrm{C}_{28}: \mathrm{C}_{20}$ & 0.06 \\
\hline triacontanoic acid, octadecyl ester & $\mathrm{C}_{30}: \mathrm{C}_{18}$ & 0.02 \\
\hline \multicolumn{3}{|l|}{ wax $\mathrm{C}_{49}$} \\
\hline eicosanoic acid, nonacosyl ester & $\mathrm{C}_{20}: \mathrm{C}_{29}$ & 0.13 \\
\hline \multicolumn{3}{|l|}{ wax $C_{50}$} \\
\hline hexadecanoic acid, tetratriacontyl ester & $\mathrm{C}_{16}: \mathrm{C}_{34}$ & 0.06 \\
\hline octadecanoic acid, dotriacontyl ester & $\mathrm{C}_{18}: \mathrm{C}_{32}$ & 0.07 \\
\hline eicosanoic acid, triacontyl ester & $\mathrm{C}_{20}: \mathrm{C}_{30}$ & 1.27 \\
\hline docosanoic acid, octacosyl ester & $\mathrm{C}_{22}: \mathrm{C}_{28}$ & 1.36 \\
\hline tetracosanoic acid, hexacosyl ester & $\mathrm{C}_{24}: \mathrm{C}_{26}$ & 0.53 \\
\hline hexacosanoic acid, tetracosyl ester & $\mathrm{C}_{26}: \mathrm{C}_{24}$ & 0.09 \\
\hline octacosanoic acid, docosyl ester & $\mathrm{C}_{28}: \mathrm{C}_{22}$ & 0.20 \\
\hline triacontanoic acid, eicosyl ester & $\mathrm{C}_{30}: \mathrm{C}_{20}$ & 0.08 \\
\hline \multicolumn{3}{|l|}{ wax $C_{51}$} \\
\hline eicosanoic acid, hentriacontyl ester & $\mathrm{C}_{20}: \mathrm{C}_{31}$ & 0.04 \\
\hline docosanoic acid, nonacosyl ester & $\mathrm{C}_{22}: \mathrm{C}_{29}$ & 0.08 \\
\hline tetracosanoic acid, heptacosyl ester & $\mathrm{C}_{24}: \mathrm{C}_{27}$ & 0.02 \\
\hline wax $\mathrm{C}_{52}$ & & \\
\hline hexadecanoic acid, hexatriacontyl ester & $\mathrm{C}_{16}: \mathrm{C}_{36}$ & 0.05 \\
\hline octadecanoic acid, tetratriacontyl ester & $\mathrm{C}_{18}: \mathrm{C}_{34}$ & 0.03 \\
\hline eicosanoic acid, dotriacontyl ester & $\mathrm{C}_{20}: \mathrm{C}_{32}$ & 0.33 \\
\hline docosanoic acid, triacontyl ester & $\mathrm{C}_{22}: \mathrm{C}_{30}$ & 1.17 \\
\hline tetracosanoic acid, octacosyl ester & $\mathrm{C}_{24} \mathrm{C}_{28}$ & 0.71 \\
\hline hexacosanoic acid, hexacosyl ester & $\mathrm{C}_{26}: \mathrm{C}_{26}$ & 0.05 \\
\hline octacosanoic acid, tetracosyl ester & $\mathrm{C}_{28}: \mathrm{C}_{24}$ & 0.03 \\
\hline triacontanoic acid, docosyl ester & $\mathrm{C}_{30}: \mathrm{C}_{22}$ & 0.24 \\
\hline wax $\mathrm{C}_{54}$ & & \\
\hline hexadecanoic acid, octatriacontyl ester & $\mathrm{C}_{16}: \mathrm{C}_{38}$ & 0.01 \\
\hline eicosanoic acid, tetratriacontyl ester & $\mathrm{C}_{20}: \mathrm{C}_{34}$ & 0.03 \\
\hline docosanoic acid, dotriacontyl ester & $\mathrm{C}_{22}: \mathrm{C}_{32}$ & 0.14 \\
\hline tetracosanoic acid, triacontyl ester & $\mathrm{C}_{24} \mathrm{C}_{30}$ & 0.30 \\
\hline hexacosanoic acid, octacosyl ester & $\mathrm{C}_{26}: \mathrm{C}_{28}$ & 0.08 \\
\hline octacosanoic acid, hexacosyl ester & $\mathrm{C}_{28}: \mathrm{C}_{26}$ & 0.02 \\
\hline triacontanoic acid, tetracosyl ester & $\mathrm{C}_{30}: \mathrm{C}_{24}$ & 0.01 \\
\hline
\end{tabular}

guaiacol (23), 4-methylguaiacol (28), 4-ethylguaiacol (3), and trans-4-propenylguaiacol (55) were also detected, although in lower amounts. Moreover, several oxidized phenols, such as vanillin (40), guaiacylacetone (47), guaiacyl vinyl ketone (50), and trans-coniferylaldehyde (57) were also identified, together with the lignin precursor trans-coniferyl alcohol (58). The $p$-hydroxyphenyl and syringyl counterparts were also detected, although in lower amounts. The relative molar distribution of the different lignin units H:G:S was 13:53:34 with a S/G molar ratio of 0.64 . No sinapyl or coniferyl acetates have been detected in hemp fibers. These acetylated lignin units have been detected by Py-GC/MS in nonwood fibers $(16,20)$ with syringyl-rich lignins such as kenaf, yute, sisal, and abaca but were not detected in other fibers with guaiacyl-rich lignin such as flax (20). In the present work, the presence of cinnamic acids in the hemp fibers were analyzed using $\mathrm{Py}-\mathrm{GC} / \mathrm{MS}$ in the presence of TMAH. The methyl derivatives of $p$-coumaric and ferulic acids (4-methoxycinnamic acid and 3,4-dimethoxycinnamic acid methyl esters, respectively) were found in only trace amounts (cinnamic acids/lignin molar ratio of 0.04), with a predominance of ferulic acid over $p$-coumaric acid.

In general, the efficiency of pulping is directly proportional to the amounts of $\mathrm{S}$ units in lignin. The $\mathrm{G}$ units have a $\mathrm{C}-5$ aromatic position (and $\mathrm{H}$ units both $\mathrm{C}-3$ and $\mathrm{C}-5$ ) available for very strong carbon-carbon bonds, which make them fairly resistant to the pulping depolymerization. Therefore, the low S/G ratio of hemp fibers may make them more difficult to delignify (and bleach) because of the higher condensation degree of the lignin, despite having a very low lignin content. In fact, the difficulty in delignifying (and bleaching) hemp soda/ anthraquinone pulp has been revealed by the pulp mill using these fibers (personal communication).

Lipid Composition. The total lipid extract of the industrial hemp bast fibers accounted for $0.5 \%$ of the total fiber. The underivatized and silylated extracts were analyzed by GC and GC/MS. The GC/MS chromatogram of the underivatized hemp lipids is shown in Figure 2, and the detailed list with the identities and abundances of the main compounds present in the hemp extracts is summarized in Table 2 . The most predominant lipid classes in hemp fibers were series of $n$-alkanes $(29 \%)$, free (17\%) and esterified (15\%) sterols and triterpenols, $n$-fatty acids $(12 \%)$, and waxes $(12 \%)$. Minor amounts of $n$-aldehydes, $n$-fatty alcohols, and steroid and triterpenoid hydrocarbons and ketones were also present in these fibers. The series of $n$-alkanes were identified in the range from $\mathrm{C}_{21}$ to $\mathrm{C}_{33}$ with a strong odd-over-even carbon atom number predominance, nonacosane $\left(\mathrm{C}_{29}\right)$ being the most predominant. $n$-Alkanes with even carbon atom numbers $\left(\mathrm{C}_{26}, \mathrm{C}_{28}\right.$, and $\left.\mathrm{C}_{30}\right)$ were also identified.

Sterols and triterpenols were also present among the lipids of hemp fibers. Sitosterol was the most abundant among the free sterols with the presence of minor amounts of stigmastanol, stigmasterol, and campesterol. Lower amounts of sitosterol could also be found in ester form. Several steryl glycosides, such as sitosteryl, campesteryl, and stigmasteryl 3- $\beta$-D-glucopyranosides, were also identified in minor amounts, the former being the most predominant. The identification of steryl glycosides was accomplished (after BSTFA derivatization of the lipid extract) by comparison with the mass spectra and relative retention times of authentic standards (23). Among triterpenols, $\beta$-amyrins predominated in both free and esterified form, and $\alpha$-amyrins were also present. Significant amounts of triterpenol acetates (including $\beta$ - and $\alpha$-amyrin, taraxerol, and lupeol acetates) were identified in the hemp fiber extracts. Several steroid hydrocar- 
bons (such as stigmasta-3,5-diene and stigmasta-3,5,22-triene) and steroid and triterpenoid ketones (such as stigmast-4-en-3one, stigmastan-3-one, stigmasta-7,22-dien-3-one, stigmastane3,6-dione and $\beta$ - and $\alpha$-amyrenones) were also identified.

Free fatty acids were present in the range from tetradecanoic $\left(\mathrm{C}_{14}\right)$ to dotriacontanoic $\left(\mathrm{C}_{32}\right)$ acids, with a strong even-overodd carbon atom predominance. Palmitic $\left(\mathrm{C}_{16: 0}\right)$ and linoleic $\left(\mathrm{C}_{18: 2}\right)$ acids were the most abundant followed by oleic $\left(\mathrm{C}_{18: 1}\right)$ and stearic $\left(\mathrm{C}_{18: 0}\right)$ acids. $\alpha$-Hydroxy fatty acids were also identified among the hemp extractives, and the series was present in the range from 2-hydroxyhexadecanoic acid to 2-hydroxypentacosanoic acid.

Waxes were also important components of the hemp fiber extracts and were found in the range from $\mathrm{C}_{40}$ to $\mathrm{C}_{54}$ with a strong predominance of the even carbon atom number homologues, the $\mathrm{C}_{44}$ and $\mathrm{C}_{46}$ analogues being the most abundant. Among the waxes, the GC/MS analysis revealed that each chromatographic peak consisted of a complex mixture of different long-chain fatty acids esterified to different long-chain fatty alcohols. The identification and quantitation of the individual long-chain esters in each chromatographic peak were resolved on the basis of the mass spectra of the peaks. The mass spectra of long-chain esters are characterized by a base peak produced by a rearrangement process involving the transfer of $2 \mathrm{H}$ atoms from the alcohol chain to the acid chain, giving a protonated acid ion $(24,25)$. Therefore, the base peak gives the number of carbon atoms in the acid moiety and the molecular ion the total number of carbon atoms in the ester. It is possible then to determine the individual contribution of esters to every chromatographic peak by mass spectrometric determination of the molecular ion and the base peak. Quantitation of individual esters was accomplished by integrating areas in the chromatographic profiles of ions characteristic for the acidic moiety. The detailed structural composition and abundance of the high molecular weight waxes identified in the hemp fiber are shown in Table 3. The esterified fatty acids ranged from $\mathrm{C}_{16}$ to $\mathrm{C}_{30}$ and the esterified fatty alcohols from $\mathrm{C}_{16}$ to $\mathrm{C}_{38}$. The acyl moiety of the waxes was exclusively constituted by saturated fatty acids with even carbon number, docosanoic $\left(C_{22}\right)$ and eicosanoic $\left(C_{20}\right)$ acids being the most predominant. Waxes with unsaturated fatty acids could not be detected, despite the high amounts of oleic and linoleic acids being present in free form. Among the esterified alcohols, the most predominant was docosanol $\left(\mathrm{C}_{22}\right)$, followed by octacosanol $\left(\mathrm{C}_{28}\right)$, hexacosanol $\left(\mathrm{C}_{26}\right)$, triacontanol $\left(\mathrm{C}_{30}\right)$, and tetracosanol $\left(\mathrm{C}_{24}\right)$, although they showed the presence of low amounts of the odd carbon atom number homologues, which accounted for the odd carbon atom number waxes. The predominant wax was $\mathrm{C}_{46}$, mostly constituted by eicosanoic acid $\left(\mathrm{C}_{20}\right)$ esterified to hexacosanol $\left(\mathrm{C}_{26}\right)$ and by docosanoic acid $\left(\mathrm{C}_{22}\right)$ esterified to tetracosanol $\left(\mathrm{C}_{24}\right)$.

$n$-Fatty alcohols ranging from $\mathrm{C}_{18}$ to $\mathrm{C}_{32}$ were present in the hemp extracts with the presence of only the even carbon atom homologues, triacontanol $\left(\mathrm{C}_{30}\right)$ and octacosanol $\left(\mathrm{C}_{28}\right)$ being the most abundant. It can be observed that the series of free fatty alcohols parallels the distribution of the esterified fatty alcohols in waxes. A series of $n$-aldehydes ranging from $\mathrm{C}_{21}$ to $\mathrm{C}_{32}$ were identified in the hemp fibers with triacontanal $\left(\mathrm{C}_{30}\right)$ and octacosanal $\left(\mathrm{C}_{28}\right)$ predominating. The distribution of the aldehyde series correlates to that of free alcohols, as usually occurs in the plant kingdom, suggesting that aldehydes are intermediates in the biosynthesis of alcohols $(26,27)$.

The different lipid classes have different behaviors during cooking. The waxes are hydrolyzed during alkaline cooking, and the fatty acids are dissolved. At sufficiently high $\mathrm{pH}$ (as in alkaline pulping), the acids dissociate and form fatty acid soaps and can thus dissolve in water to quite a high extent, forming fatty acid soaps. On the other hand, alkanes, fatty alcohols, sterols and triterpenols, steroid hydrocarbons and ketones, and steryl glycosides survive cooking. These compounds have a very low solubility in water and are difficult to remove and, therefore, can be at the origin of pitch problems. In conclusion, the present work reports the chemical composition of lignin and lipids of industrial hemp fibers. Knowledge of the chemical composition of the main components of hemp fibers will be useful for a better utilization of nonwood plants.

\section{ACKNOWLEDGMENT}

We thank CELESA (Tortosa, Spain) for providing the hemp bast fibers.

\section{LITERATURE CITED}

(1) Moore, G. Nonwood Fibre Applications in Papermaking; Pira International: Leatherhead, Surrey, U.K., 1996.

(2) Struik, P. C.; Amaducci, S.; Bullard, M. J.; Stutterheim, N. C.; Venturi, G.; Cromack, H. T. H. Agronomy of fibre hemp (Cannabis sativa L.) in Europe. Ind. Crop Prod. 2000, 11, 107118.

(3) del Río, J. C.; Gutiérrez, A.; Hernando, M.; Landín, P.; Romero, J.; Martínez, A. T. Determining the influence of eucalypt lignin composition in paper yield using Py-GC/MS. J. Anal. Appl. Pyrol. 2005, 74, 110-115.

(4) González-Vila, F. J.; Almendros, G.; del Río, J. C.; Martín, F.; Gutiérrez, A.; Romero, J. Ease of delignification assessment of wood from different Eucalyptus species by pyrolysis (TMAH)GC/MS and CP/MAS ${ }^{13} \mathrm{C}-\mathrm{NMR}$ spectroscopy. J. Anal. Appl. Pyrol. 1999, 49, 295-305.

(5) Hillis, W. E.; Sumimoto, M. Effect of extractives on pulping. In Natural Products of Woody Plants II; Rowe, J. W., Ed.; Springer-Verlag: Berlin, Germany, 1989; pp 880-920.

(6) Back, E. L.; Allen, L. H. Pitch Control, Wood Resin and Deresination; Tappi Press: Atlanta, GA, 2000.

(7) del Río, J. C.; Gutiérrez, A.; González-Vila, F. J.; Martín, F.; Romero, J. Characterization of organic deposits produced in the kraft pulping of Eucalyptus globulus wood. J. Chromatogr. A 1998, 823, 457-465.

(8) Gutiérrez, A.; del Río, J. C. Chemical characterization of pitch deposits produced in the manufacturing of high-quality paper pulps from hemp fibers. Bioresour. Technol. 2005, 96, 14451450.

(9) Gutiérrez, A.; del Río, J. C.; Martínez, M. J.; Martínez, A. T. The biotechnological control of pitch in paper pulp manufacturing. Trends Biotechnol. 2001, 19, 340-348.

(10) Ali, M.; Sreekrishnan, T. R. Aquatic toxicity from pulp and paper mill effluents: a review. Adv. Environ. Res. 2001, 5, 175-196.

(11) Faix, O.; Meier, D.; Fortmann, I. Thermal degradation products of wood. A collection of electron-impact (EI) mass spectra of monomeric lignin derived products. Holz Roh-Werkst. 1990, 48, $351-354$.

(12) Ralph, J.; Hatfield, R. D. Pyrolysis-GC/MS characterization of forage materials. J. Agric. Food Chem. 1991, 39, 1426-1437.

(13) Gutiérrez, A.; del Río, J. C.; González-Vila, F. J.; Martín, F. Analysis of lipophilic extractives from wood and pitch deposits by solid-phase extraction and gas chromatography. J. Chromatogr. A 1998, 823, 449-455.

(14) Technical Association of the Pulp and Paper Industry. Test Methods, 1992-1993; TAPPI: Atlanta, GA, 1993.

(15) Bocchini, P.; Galletti, G. C.; Camarero, S.; Martínez, A. T. Absolute quantitation of lignin pyrolysis products using an internal standard. J. Chromatogr. A 1997, 773, 227-232.

(16) Gutiérrez, A.; Rodríguez, I. M.; del Río, J. C. Chemical characterization of lignin and lipid fractions in kenaf bast fibers used for manufacturing high-quality papers. J. Agric. Food Chem. 2004, 52, 4764-4773. 
(17) van Dam, J. E. G.; van Vilsteren, G. E. T.; Zomers, F. H. A.; Shannon, W. B.; Hamilton, I. T. Increased Application of Domestically Produced Plant Fibres in Textiles, Pulp and Paper Production and Composite Materials; Directorate-General XII, Science, Research and Development, European Commission: Brussels, Belgium, 1994.

(18) Gutiérrez, A.; del Río, J. C. Lipids from flax fibers and their fate in alkaline pulping. J. Agric. Food Chem. 2003, 51, 49654971.

(19) Gutiérrez, A.; del Río, J. C. Lipids from flax fibers and their fate in alkaline pulping. J. Agric. Food Chem. 2003, 51, 69116914 (erratum).

(20) del Río, J. C.; Gutiérrez, A.; Martínez, A. T. Identifying acetylated lignin units in non-wood fibers using pyrolysis-gas chromatography/mass spectrometry. Rapid Commun. Mass Spectrom. 2004, 18, 1181-1185.

(21) Nimz, H. Beech lignin-proposal of a constitutional scheme. Angew. Chem., Int. Ed. 1974, 13, 313-321.

(22) Adler, E. Lignin chemistry-past, present and future. Wood Sci. Technol. 1977, 11, 169-218.

(23) Gutiérrez, A.; del Río, J. C. Gas chromatography/mass spectrometry demonstration of steryl glycosides in eucalypt wood,
Kraft pulp and process liquids. Rapid Commun. Mass Spectrom. 2001, 15, 2515-2520.

(24) Sharkey, Jr., A. G.; Shultz, J. L.; Friedel, R. A. Mass spectra of esters. Formation of rearrangement ions. Anal. Chem. 1959, 31 , 87-94.

(25) Moldovan, Z.; Jover, E.; Bayona, J. M. Systematic characterisation of long-chain aliphatic esters of wool wax by gas chromatography-electron impact ionisation mass spectrometry. J. Chromatogr. 2002, 952, 193-204.

(26) Tulloch, A. P. Chemistry of waxes of higher plants. In Chemistry and Biochemistry of Natural Waxes; Kolattukudy, P. E., Ed.; Elsevier: Amsterdam, The Netherlands, 1976; pp 236-252.

(27) Bianchi, G.; Plant waxes. In Waxes: Chemistry, Molecular Biology and Functions; Hamilton, R. J., Ed.; The Oily Press: Dundee, Scotland, 1995; pp 175-222.

Received for review November 24, 2005. Revised manuscript received January 16, 2006. Accepted January 19, 2006. This study has been supported by the Spanish Ministerio de Ciencia y Tecnología (MCyT) and FEDER funds (Projects AGL2002-00393).

JF052935A 